\title{
Adaptive Control Strategy of VSG Based on Improved Multi- objective Particle Swarm Optimization Algorithm
}

\author{
Zhen Tian ${ }^{1 *}$, Xiaojin $\mathrm{Fu}^{1}$, Jing $\mathrm{Lv}^{1}$ and Hongyan $\mathrm{Zhu}^{1}$ \\ ${ }^{1}$ School of Mechanical Engineering, Shanghai Dianji University, Shanghai, 201306, China
}

\begin{abstract}
In order to solve the problem that the frequency stability of power system is threatened due to inertia and damping reduction. In this paper, an adaptive control strategy of virtual synchronous generator based on improved multi-objective particle swarm optimization algorithm with physical process is proposed. This method takes into account the static and dynamic performance of the system, and in the face of small disturbances, it can adjust the moment of inertia and damping in a planned way and reduce the search range of the moment of inertia, thereby reducing the frequency deviation and speeding up the end of the transition process.
\end{abstract}

\section{Introduction}

With the rapid development of new energy generation, the moment of inertia and damping ratio in power system decrease, which affects the frequency stability of power system. The control strategy of virtual synchronous generator (VSG) makes the inverter have inertia and damping characteristics by introducing the rotor motion equation of synchronous generator, thus enhancing the frequency stability of the system.

The concept of VSG is proposed in reference [1]. In reference [2], VSG is used in microgrid, but the moment of inertia and damping are both fixed values. When the load or generated active power changes, it will lead to large frequency deviation and long transition time. In literature [3], an adaptive rotational inertia control strategy of VSG based on particle swarm optimization is proposed, but the influence of damping on frequency is neglected. In reference [4], the frequency variation process of VSG under small disturbance is analyzed, and the influence of moment of inertia and damping on frequency is summarized, but the range of parameters is not given.

In this paper, an adaptive control strategy of VSG based on improved multi-objective particle swarm optimization algorithm is proposed. This method can dynamically adjust the moment of inertia and damping value, thereby reducing the frequency deviation of the system, and the objective function 2 can ensure that the system has a relatively good static performance and dynamic performance. Finally, the simulation results of adopting non-adaptive control with recommended fixed values of moment of inertia and damping, adaptive control, and adaptive control strategy based on improved multi-objective particle swarm optimization algorithm are compared respectively by using Simulink. The results show that the adaptive control strategy of VSG based on the improved multi-objective particle swarm algorithm has the best control effect.

\section{Principles of VSG}

\subsection{Influence of $J$ and $D$ on active power}

Equation (1) is the motion equation of VSG rotor, which is the core part of VSG, and provides rotational inertia and damping coefficient for inverter operation, thus increasing the frequency stability of the system.

$$
\left\{\begin{array}{l}
T_{\mathrm{m}}-T_{\mathrm{e}}=J \frac{d \omega}{d t}+D\left(\omega-\omega_{\mathrm{N}}\right) \\
\frac{d \theta}{d t}=\omega-\omega_{\mathrm{N}}
\end{array}\right.
$$

In which: $T_{\mathrm{m}}$ is mechanical torque $(\mathrm{N} \cdot \mathrm{m}) ; T_{\mathrm{e}}$ is electromagnetic torque $(\mathrm{N} \cdot \mathrm{m}) ; J$ is moment of inertia $(\mathrm{kg} \cdot \mathrm{m} 2) ; D$ is damping coefficient $(\mathrm{N} \cdot \mathrm{m} \cdot \mathrm{s} / \mathrm{rad})$.

Assuming that the generator rotor pole pair $\mathrm{p}=1$, the electrical angle $\omega$ is equal to the mechanical angular velocity $\Omega$. Therefore, when the rotor rotates at the rated speed $\omega_{\mathrm{N}}$, the kinetic energy stored on the rotor is:

$$
W_{\mathrm{K}}=\frac{1}{2} J \omega_{\mathrm{N}}^{2}
$$

The moment of inertia of VSG can be determined by equation (3).

$$
T_{\mathrm{J}}=\frac{2 W_{\mathrm{K}}}{\mathrm{S}_{\mathrm{N}}}
$$

$T_{\mathrm{J}}$ is the inertia time constant of the generator (s), which indicates the time it takes for the generator to go from static state to rated speed under rated torque; The inertia time parameter $T_{\mathrm{J}}$ of the generator is generally 
$2 \sim 10$ s. The inertia time constant of VSG is generally $3 \sim 12 \mathrm{~s} . T_{\mathrm{J}}=5$ is usually taken in engineering.
Figure 1 is the VSG virtual inertia controller model diagram.

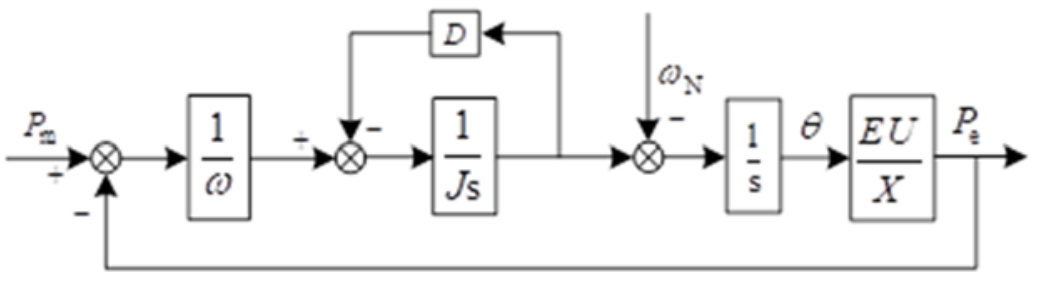

Figure 1. Model diagram of VSG virtual inertia controller

In the figure, $E$ and $U$ are phase electromotive force and phase voltage of phase a respectively, and $X$ is equivalent inductive reactance of VSG. In order to further explore the influence of $J$ and $D$ on frequency stability, this problem can be studied by obtaining the transfer function between electromagnetic power and mechanical power.

When the power angle $\theta$ is relatively small, $\sin \theta$ and $\theta$ are approximately equal, so:

$$
P_{\mathrm{e}}=\frac{3 E U}{X} \sin \theta=\frac{3 E U}{X} \theta
$$

Therefore, the transfer function of VSG virtual inertia controller can be obtained as follows:

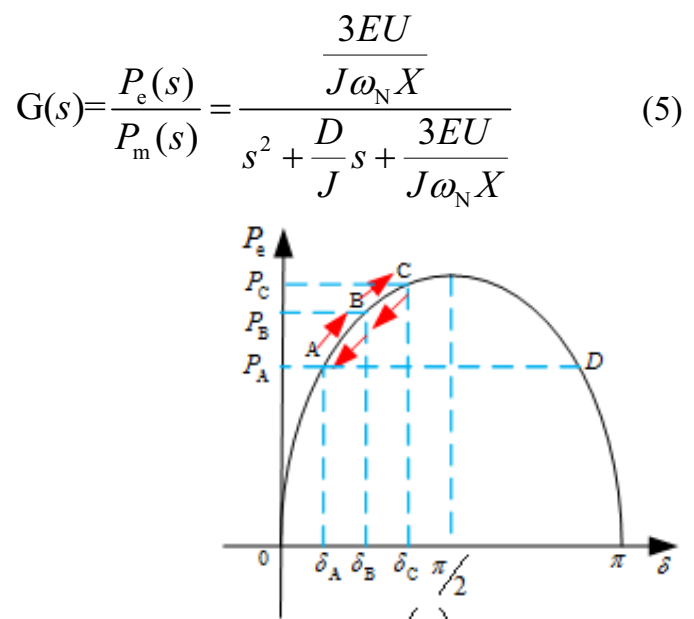

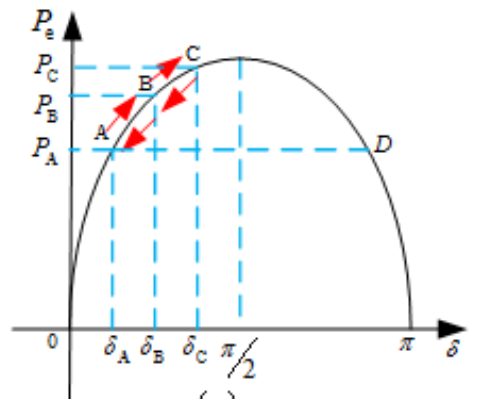

(a)
The natural oscillation angular frequency $\omega_{\mathrm{n}}$ and damping ratio $\zeta$ can be obtained by formula (6):

$$
\left\{\begin{array}{l}
\omega_{\mathrm{n}}=\sqrt{\frac{3 E U}{J \omega_{\mathrm{n}} X}} \\
\xi=\frac{D}{2} \sqrt{\frac{\omega_{\mathrm{n}} X}{3 J E U}}
\end{array}\right.
$$

\subsection{Variation rules of $J$ and $D$ under small disturbance}

Figure 2(a) and 2(b) show the power angle characteristic curve and rotor swing curve of synchronous generator respectively, and the two curves correspond to each other one by one.

Figure 2(a). power angle characteristic curve. Figure 2(b). rotor swing curve.

$t_{1} \sim t_{2}$ : At $t_{1}$, the input power increases from $P_{\mathrm{A}}$ to $P_{\mathrm{B}}$. Since the mechanical torque is greater than the electromagnetic torque at this time, the rotor will be accelerated by the unbalanced torque, and the rate of change of the rotor angular velocity $d \omega / d t>0$. Unbalanced torque decreases gradually, that is, angular acceleration $d \omega / d t$ decreases gradually. Until $t_{2}$, $d \omega / d t$ decreases to 0 , at which time $\omega$ reaches the maximum. In this process, the deviation of rotor acceleration and angular frequency should be smaller, so $J$ and $D$ should be increased appropriately.

$t_{2} \sim t_{3}$ : When the electromagnetic power is greater than the mechanical power, the angular acceleration $d \omega / d t<0$ and angular velocity $\omega$ of the rotor gradually decrease until $\omega$ decreases to $\omega_{\mathrm{N}}$, and then the power angle reaches the maximum $\delta_{\mathrm{C}}$. In this process, we hope that the angular velocity $\omega$ can return to $\omega_{\mathrm{N}}$ as soon as possible. Therefore, $J$ should choose smaller, $D$ should choose appropriate larger.

$t_{3} \sim t_{4}: d \omega / d t<0$, $\omega$ will decrease further. In this process, it is expected that the deviation between the absolute value of angular acceleration of rotor and angular velocity of rotor will be small. Therefore, $J$ and $D$ should be appropriately selected larger.

$t_{4} \sim t_{5:} d \omega / d t>0$, $\omega$ will gradually increase. In the process, $\omega$ should return $\omega_{\mathrm{N}}$ as soon as possible. Therefore, $J$ should be chosen smaller, and $D$ should be 
chosen appropriately larger.

This process can be summarized in Table 1 .

Table 1. Changes of various parameters under small disturbances and selection rules of $\mathrm{J}$ and $\mathrm{D}$

\begin{tabular}{ccccc}
\hline period & $d \omega / d t$ & $\Delta \omega$ & The changing trend of $J$ & The changing trend of $D$ \\
\hline$t_{1} \sim t_{2}$ & $>0$ & $>0$ & Appropriate increase & Appropriate increase \\
$t_{2} \sim t_{3}$ & $<0$ & $>0$ & Appropriate reduction & Appropriate increase \\
$t_{3} \sim t_{4}$ & $<0$ & $<0$ & Appropriate increase & Appropriate increase \\
$t_{4} \sim t_{5}$ & $>0$ & $<0$ & Appropriate reduction & Appropriate increase \\
\hline
\end{tabular}

\section{Improved multi-objective particle swarm optimization}

Particle Swarm Optimization (PSO) simulated the foraging process of birds. The basic attributes of PSO are position and speed. They fly at a certain speed in the solution space, share position information with each other, and constantly adjust their own flight direction according to the historical best position $P_{\text {best }}$ of the particle itself and the best position $g_{\text {best }}$ found by the whole group. In the whole optimization process, the position of each flight update may become the solution of the problem.

Assuming that the solution space is D-dimensional and the population number is $n$, the position of the particle $i$ can be written as: $X_{i}=\left(x_{i 1}, x_{i 2}, \cdots, x_{i D}\right)$, $i=1,2, \cdots, N$; Velocity of the particle $i$ can be written as: $V_{\mathrm{i}}=\left(v_{i 1}, v_{i 2}, \cdots, v_{i D}\right), i=1,2, \cdots N$; Individual best position of the $i^{\text {th }}$ particle can be written as: $p_{\text {best }}=\left(p_{i 1}, p_{i 2}, \cdots, p_{i D}\right) \quad, \quad i=1,2, \cdots, N \quad ; \quad$ Optimal location of group can be written as: $g_{\text {best }}=\left(p_{g 1}, p_{g 2}, \cdots, p_{g D}\right), i=1,2, \cdots, N$;

In the iterative process of particle swarm optimization, the fitness function is used to evaluate whether the positions of individuals and groups are the best positions of individuals $P_{\text {best }}$ and best positions $g_{\text {best }}$ of groups, and the speed and position are constantly updated according to the speed update formula (7) and position update formula (8).

$$
v_{i j}(t+1)=\omega v_{i j}(t)+c_{1} r_{1}\left(p_{i j}(t)-x_{i j}(t)\right)+c_{2} r_{2}\left(g_{i j}(t)-x_{i j}(t)\right)
$$

$$
x_{i j}(t+1)=x_{i j}(t)+v_{i j}(t+1)
$$

In which: $v_{i j}(t)$ is he velocity of the $i^{\text {th }}$ particle in the $j^{\text {th }}$ dimension in the $\mathrm{t}$ generation; $\omega$ is Inertial weight coefficient; $c_{1}$ and $c_{2}$ is learning factor; $r_{1}$ and $r_{2}$ is random numbers in $[0,1] ; p_{i j}(t)$ is the individual best position of the $i^{\text {th }}$ particle in the $j^{\text {th }}$ dimension in the $\mathrm{t}$ generation. $g_{i j}(t)$ is the position where the fitness of all particles is the best in the $t$ generation, that is, the best position of the group; $x_{i j}(t)$ is the current position of the $i^{\text {th }}$ particle in the $j^{\text {th }}$ dimension in the $t$ generation.

When $\zeta=0.707$, the system can obtain both good static performance and good dynamic performance. Therefore, two objective functions can be set. The first objective function should be able to ensure that the frequency deviation of VSG is as small as possible under small disturbance. Objective function 2 is used to make the system have good comprehensive performance. The first objective function should be a function with the rotational inertia $J$ and damping coefficient $D$ as independent variables and the frequency (angular frequency) as dependent variables. It can be concluded from equation (1):

$$
f_{1}(J, D)=|\Delta \omega|=\left|\frac{T_{\mathrm{m}}-T_{\mathrm{e}}}{D}\left(1-\mathrm{e}^{-\frac{D}{J} t}\right)\right|
$$

Objective function 2 should be used to measure the proximity of $\zeta$ to 0.707 , which can be obtained from equation (6):

$$
f_{2}(J, D)=\left|\frac{D}{2} \sqrt{\frac{\omega_{\mathrm{n}} X_{\mathrm{L}}}{3 J E U}}-0.707\right|
$$

There are two ways to deal with objective function in multi-objective algorithm. The first one is to transform multiple objective problems into single objective problems by setting the weights of functions, i.e. $F=\lambda_{1} f_{1}+\lambda_{2} f_{2}+\cdots$, where $\lambda_{1}$ and $\lambda_{2}$ are weight coefficients. The second method is the non-inferior solution set method, which can obtain the non-inferior solutions of multiple objective functions, and then select the most appropriate group of solutions among the multiple non -inferior solutions.

Equation (9) is a typical negative exponential function with good convergence characteristics. However, it is found in experiments that this function is easy to mature prematurely and fall into a local optimal solution. Scheme 1, although it is simple to transform multiobjective problems into single-objective problems, can only get a set of solutions, and it is impossible to determine whether the solutions are local optimal solutions. In order to obtain a reliable solution, this paper uses the Pareto non-inferior set method to obtain multiple sets of solutions, and select the best solution from them.

At the same time, in order to solve the problem that the objective function is easy to precocious in the calculation process, this paper introduces the sorting selection method and crossover and mutation operation in genetic algorithm to increase the population diversity.

The following is the basic process of multi-objective particle algorithm:

(1) Set iteration times, population size, speed boundary and position boundary.

(2) Initialize the position and velocity of particles.

(3) Calculate the particle fitness value.

(4) Find out the best fitness value and its corresponding position. At the same time, take the position of the first generation particles as the individual 
best position and the original non-inferior solution set, sort the non-inferior solution set according to distance, and take the position of the third farthest particle as the best position of the group.

(5) Update the speed and position of particles, the speed and position should be limited according to the constraint conditions. When the upper limit or lower limit value is exceeded, it will be limited to the limit value.

(6) The fitness of each particle in the iterative process is calculated and compared with the individual fitness value of the previous iteration, so as to find out the best fitness value of particles and the best position of individuals.

(7) Comparing the individual best position with the non-inferior solution set, updating the non-inferior solution set and the group best position, and performing cross mutation operation on the non-inferior set. The mutation rate of $10 \%$ is set in this paper, of which $6 \%$ are genetic mutations and $4 \%$ are chromosomal crossovers.

(8) Judge whether the termination conditions are met. If the conditions are met, the entropy weight method is used for comprehensive evaluation, and the particle positions corresponding to the minimum entropy value are assigned to $\mathrm{J}$ and $\mathrm{D}$ respectively. Otherwise, repeat steps (5) (7).
(9) Judging whether small disturbance is encountered and the disturbance is greater than the action dead zone, and whether $\Delta \omega(d \omega / d t)$ is greater than 0 or less than 0 .

(10) If (8) is satisfied, and; Then the population search range of particles is reduced, the linearly decreasing inertia weight is changed to a smaller fixed weight, and the flight speed of particles is reduced, and the original solved value of $\mathrm{J}$ is taken as the lower limit of the search range. If $\Delta \omega(d \omega / d t)<0, \mathrm{~J}$ is regarded as the upper limit of particle search range.

(11) In the iterative optimization process, since the electrical quantity changes quickly, the result will be output after the number of iterations $1 \sim 2$, and return to step (9) for judgment.

\section{$4 \quad$ Simulation verification and result analysis}

In order to verify the effectiveness of the proposed method, the simulation model under the grid-connected mode is built in Simulink. The following table shows the parameters used in simulation.

Table 2. The main parameters of simulation

\begin{tabular}{cccc}
\hline Parameters & Value & Parameters & Value \\
\hline DC voltage $U_{\mathrm{dc}} / \mathrm{V}$ & 700 & Active power reference value $\mathrm{P}_{\mathrm{ref}} / \mathrm{kW}$ & 20 \\
Switching frequency $f_{\mathrm{c}} / \mathrm{kHz}$ & 20 & Reactive power reference value $\mathrm{Vref} / \mathrm{kVar}$ & 0 \\
Rated frequency $f_{0} / \mathrm{Hz}$ & 50 & Virtual rotational inertia $J / \mathrm{kg} \cdot \mathrm{m} 2)$ & 1 \\
Sampling time $\mathrm{T}_{\mathrm{sp}} / \mu \mathrm{s}$ & 2.5 & Virtual damping coefficient $\mathrm{D} / \mathrm{N} \cdot \mathrm{m} \cdot \mathrm{s} / \mathrm{rad})$ & 40 \\
Filtering inductance $L_{\mathrm{l}} / \mathrm{mH}$ & 4 & Virtual resistance $R_{\mathrm{s}} / \Omega$ & -1 \\
Equivalent resistance $R_{\mathrm{l}} / \Omega$ & 0.2 & Virtual inductance $L_{\mathrm{s}} / \mathrm{mH}$ & 0.4 \\
Filter capacitance $\mathrm{C} / \mu \mathrm{F}$ & 20 & Proportional coefficient of current loop $K_{\mathrm{cp}}$ & 66.92 \\
Inductance of line $\mathrm{L}_{2} / \mathrm{mH}$ & 1.714 & Integral coefficient of current loop $K_{\mathrm{CI}}$ & 438650 \\
Resistance of line $R_{2} / \Omega$ & 0.1 & Proportional coefficient of voltage loop $K_{\mathrm{vp}}$ & 0.2 \\
Active droop coefficient $(\mathrm{p} . \mathrm{u}$. $) K_{\mathrm{f}}$ & 20 & Integral coefficient of voltage loop $K_{\mathrm{CI}}$ & 671.74 \\
Reactive droop coefficient $(\mathrm{p} . \mathrm{u}.) K_{\mathrm{v}}$ & 20 & & \\
\hline
\end{tabular}

From equation (3), $J=0.61 \sim 2.43 \mathrm{~kg} \cdot \mathrm{m}^{2}$ can be solved. By substituting the result into equation (6) and making $\zeta=0.707$, we can get $D=32 \sim 63 \mathrm{~N} \cdot \mathrm{m} \cdot \mathrm{s} / \mathrm{rad}$. The two ranges are regarded as the range of group position of particle swarm respectively. The maximum velocity of particles should be chosen appropriately, neither too large nor too small. In this paper, the maximum velocity and minimum velocity in $J$ direction are 0.03 and -0.03 respectively; The maximum velocity and minimum velocity in $D$ direction are 0.5 and -0.5 respectively; The population number is set to 30 ; The maximum number of iterations is set to 50; Learning factor $\mathrm{C}_{1}=\mathrm{C}_{2}=1.49445$; The maximum inertia weight coefficient is set to 0.9 and the minimum value is set to 0.4. In the case of small disturbance, the local search ability of particles should be guaranteed, and the inertia weight of linear decreasing should be changed to fixed weight value of 0.4 , and the maximum and minimum velocities of particles are reduced. The maximum speed and minimum speed in $J$ direction are changed to 0.01 and -0.01 respectively, while the maximum speed and minimum speed in $D$ direction are set to 0.25 and -0.25 respectively. The number of population remained unchanged at 30 , and the maximum number of iterations decreased to one.

In the simulation, it is necessary to transmit data between Matlab and Simulink in real time. In this paper, S-function system function is used for real-time communication. Matlab itself is not a real-time simulation software, but the S-function will transfer the data into the base workspace of Matlab at the sampling time of simulation. Therefore, multi-objective particle swarm optimization can read the simulation data of Simulink from Matlab and return the calculated data to the Simulink simulation model.

In the grid connected mode, the inverter generates $15 \mathrm{kw}$ of active power in 0 seconds, increases $5 \mathrm{~kW}$ of active power in $0.8 \mathrm{~s}$ due to environmental changes or power system needs, and reduces $3 \mathrm{KW}$ of active power in $1.5 \mathrm{~s} . J$ and $D$ respectively take the moment of inertia corresponding to the inertia time constant equal to 5 seconds and the virtual damping coefficient corresponding to $\zeta=0.707$. There is no obvious difference 
in output voltage and load current curve between adaptive control based on improved multi-objective

particle swarm optimization algorithm and non-adaptive control. Only one curve is listed here.
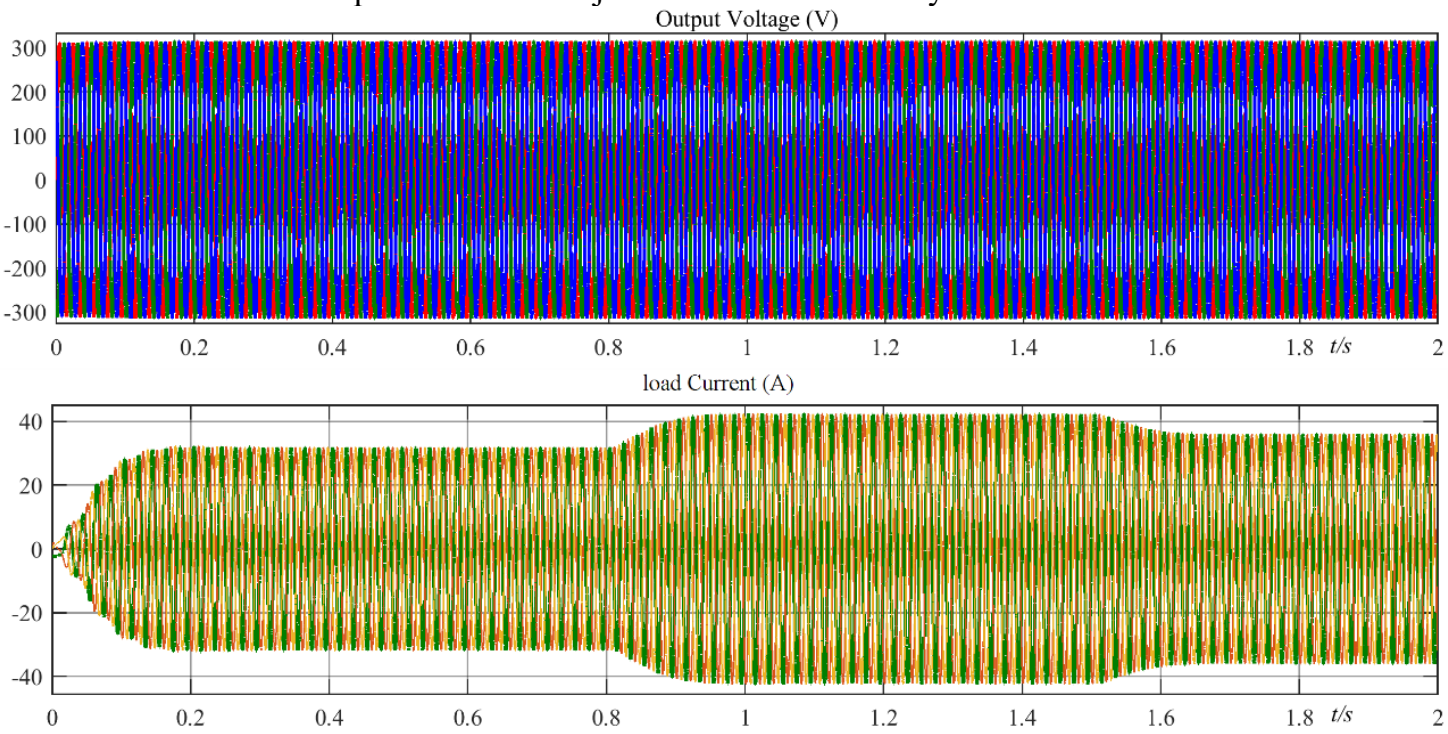

Figure 3. Output voltage and load current curves of inverter

Figure 4 shows the curves of frequency of inverter output voltage under different control modes. It can be seen from the figure that when $J$ and $D$ are the recommended values, although the frequency of the system has always met the requirements of the national standard, the frequency deviation of VSG voltage with adaptive control is smaller. When $D=60$ and $J$ adopts adaptive control, although the damping effect of oscillation is better, because $\zeta$ is the largest, the damping ratio of the control system is the largest, the adjustment time of the system is the longest, and the effect of suppressing the fluctuation of output voltage frequency is better than that of adopting adaptive control of $J$ and $D$ parameters. Comprehensive comparison shows that the adaptive control method with $J$ and $D$ parameters has the best effect, the maximum deviation of frequency is obviously small, and the time to reach steady state is obviously shortened.

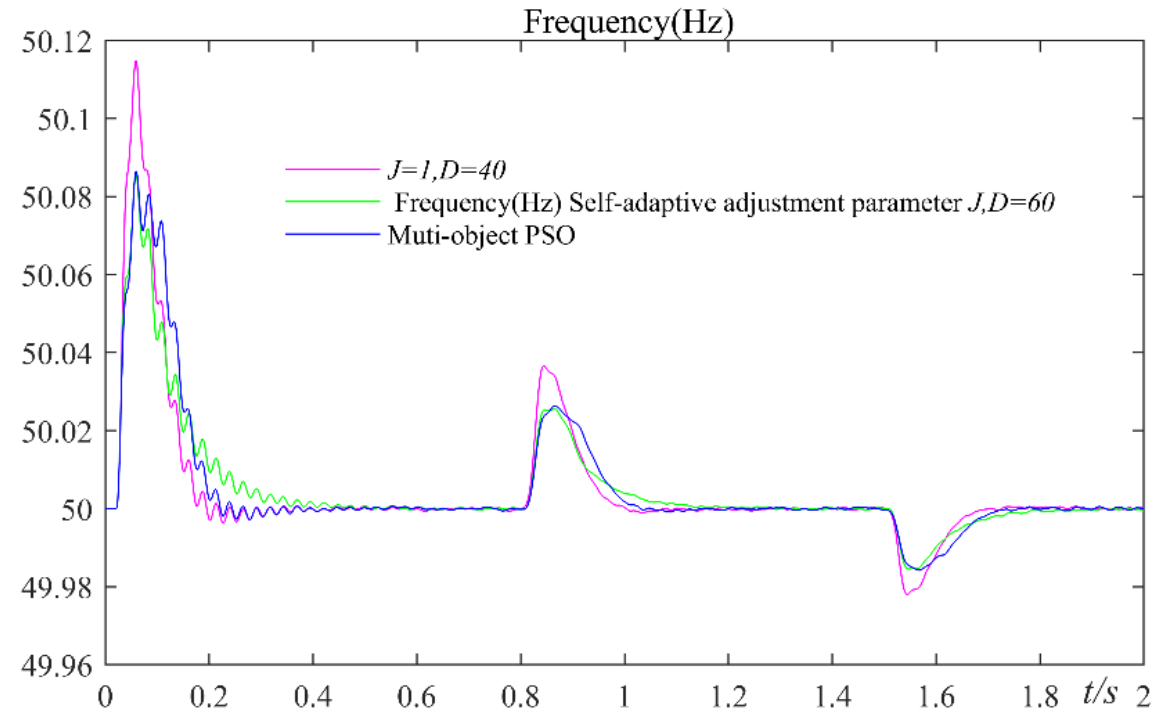

Figure 4. Curves of frequency of inverter output voltage under different control modes

\section{Conclusion}

In this paper, the principle of adaptive control is obtained by analyzing the power angle characteristic curve and swing curve of generator under small disturbance. Two objective functions are obtained by the motion equation of rotor and the function of damping ratio $\zeta$ of transfer function of active power. These two objective functions are used to ensure that the system has a small frequency deviation and a good comprehensive performance. Aiming at the problems of objective function 1, the algorithm is improved and this physical process is introduced into the algorithm. Finally, the simulation is carried out on Simulink. The experimental results show that the adaptive control strategy of VSG based on the 
improved multi-objective particle swarm optimization algorithm can effectively reduce the frequency deviation of the system, and to some extent slow down the process of frequency deviation from the rated value, and accelerate the process of frequency recovery to the rated value.

\section{References}

1. Driesen and K. Visscher. (2008) Virtual synchronous generators. 2008 IEEE Power and Energy Society General Meeting - Conversion and Delivery of Electrical Energy in the 21st Century. Pittsburgh, PA. pp. 1-3. J.

2. B. Zhang, X. Zhang, Z. Song and X. Yan. (2019) Enhanced control and parameter analysis for virtual synchronous generators without PLL. The Journal of Engineering. Commun., 16: 3082-3087. J.

3. D. Li, Q. Zhu, S. Lin and X. Y. Bian. (2017) A SelfAdaptive Inertia and Damping Combination Control of VSG to Support Frequency Stability. IEEE Transactions on Energy Conversion. vol. 32, no. 1, pp. 397-398. J.

4. Fathin Saifur Rahman, Thongchart Kerdphol, Masayuki Watanabe, Yasunori Mitani. (2019) Optimization of virtual inertia considering system frequency protection scheme[J]. Electric Power Systems Research. Commun., 170: 294-302. J. 